\title{
OCV Hysteresis in Li-Ion Batteries including Two-Phase Transition Materials
}

\author{
Michael A. Roscher, ${ }^{1}$ Oliver Bohlen, ${ }^{2}$ and Jens Vetter $^{2}$ \\ ${ }^{1}$ Institute for Power Electronics and Electrical Drives (ISEA), RWTH Aachen University, 52066 Aachen, Germany \\ ${ }^{2}$ Division of Energy Storage Systems, BMW AG, 80788 Munich, Germany
}

Correspondence should be addressed to Michael A. Roscher, michael.roscher@rwth-aachen.de

Received 29 January 2011; Accepted 28 February 2011

Academic Editor: S. Gopukumar

Copyright (C) 2011 Michael A. Roscher et al. This is an open access article distributed under the Creative Commons Attribution License, which permits unrestricted use, distribution, and reproduction in any medium, provided the original work is properly cited.

The relation between batteries' state of charge (SOC) and open-circuit voltage (OCV) is a specific feature of electrochemical energy storage devices. Especially NiMH batteries are well known to exhibit OCV hysteresis, and also several kinds of lithium-ion batteries show OCV hysteresis, which can be critical for reliable state estimation issues. Electrode potential hysteresis is known to result from thermodynamical entropic effects, mechanical stress, and microscopic distortions within the active electrode materials which perform a two-phase transition during lithium insertion/extraction. Hence, some Li-ion cells including two-phase transition active materials show pronounced hysteresis referring to their open-circuit voltage. This work points out how macroscopic effects, that is, diffusion limitations, superimpose the latte- mentioned microscopic mechanisms and lead to a shrinkage of OCV hysteresis, if cells are loaded with high current rates. To validate the mentioned interaction, Li-ion cells' state of charge is adjusted to $50 \%$ with various current rates, beginning from the fully charged and the discharged state, respectively. As a pronounced difference remains between the OCV after charge and discharge adjustment, obviously the hysteresis vanishes as the target SOC is adjusted with very high current rate.

\section{Introduction}

In the recent years, lithium-ion batteries (Li-ion) became the favorable choice for most portable energy-consuming applications. The demand for high-power capable and high efficient energy storing devices spurred research activities in the field of developing battery electrodes which offer high power and energy densities, comprising long lifetime at minimum cost efforts.

Therefore, new materials, synthesis methods, and improved electrode morphologies are introduced. The electrode material composition and morphology (e.g., grain sizes an distribution) strongly affects the electric characteristics of battery cells. Beyond the utilizable capacity and the power capability, the open-circuit voltage (OCV) is influenced by micromechanic and thermodynamical processes. The OCV hysteresis is a typical phenomenon for batteries and is well documented for nickel-metal-hydride (NiMH) battery systems [1,2]. Even in Li-ion batteries, OCV hysteresis effects can be observed [2-5], which have a minor impact on battery cells' OCV for cobalt, nickel, or manganese-based cathode systems, due to the high gradient in the specific state of charge (SOC) to OCV relation. Modern Li-ion cells comprise active materials showing only a slight gradient in the SOCOCV curves. Herein the OCV hysteresis becomes a major influencing factor for reliable OCV reconstruction, which is a critical task for the model-based state estimation as a part of a battery management system (BMS).

In the following sections, firstly the special OCV characteristics of two-phase transition materials are described with respect to hysteresis phenomena. Then, the electric properties of porous electrode geometries are outlined. Herein a possible explanation for current rate impact on the occurrence of OCV hysteresis is given. Thereafter, validation test results are presented and discussed.

\section{Two-Phase Transition Lithium Insertion}

Li-ion cells including active materials which perform a twophase transition during charge and discharge are commonly known to exhibit special OCV characteristic. The lithium iron phosphate $\left(\mathrm{LiFePO}_{4}\right)$, lithium titanate $\left(\mathrm{Li}_{4} \mathrm{Ti}_{5} \mathrm{O}_{12}\right)$, 
and lithiated graphite $\left(\mathrm{LiC}_{6}\right)$ are prominent representatives of active materials performing a two-phase transition lithium insertion and extraction process. Strong interactions between the embedded lithium and the host lattice lead to a phase separation during the two-phase transition [6]. During Li insertion in a delithiated bulk material, a lithiated phase region emerges in juxtaposition to still delithiated phase regions $[7,8]$. Further Li insertion leads to a shifting barrier between the phase regions $[9,10]$. Analogously, the $\mathrm{Li}$ extraction proceeds vice versa. Due to the occurrence of at least two different material phases within, the electrode's electrochemical potential remains almost constant during lithium insertion and extraction, respectively.

That means that, through the occurrence of the twophase transition a high differential capacity at a certain electrode voltage and therefore a typical voltage plateau during charging or discharging the battery cell can be observed [11]. Figure 1 depicts the typical potential curves (against $\mathrm{Li} / \mathrm{Li}^{+}$) of the aforementioned Li-ion electrode materials, plotted over the stoichiometric lithium content $x$ [12-14].

Both the lithium iron phosphate and lithium titanate comprise one wide potential plateau between lithiated and delithiated states. In contrast, the graphite shows several potential plateaus attributed to all in all five different energetic favorable phase contents. Between the favorable lithiation states, the graphite performs two-phase transitions [12].

Beyond that, cells with two-phase active materials are documented to show pronounced OCV hysteresis [3, 4, 15, 16], where an electrode's potential after $\mathrm{Li}$ insertion is lower (against $\mathrm{Li} / \mathrm{Li}^{+}$reference) than after $\mathrm{Li}$ extraction, even with the same stoichiometric Li content. The OCV hysteresis, that is, load history-dependent OCV variation, results from various mechanisms. On one hand, different lattice constants of lithiated and delithiated phases cause mechanical stress at the phase barrier leading to potential drops inside the individual particles [17]. Moreover, lattices distortions, as a result of doping compounds, hinder the propagation of the phase barrier and therefore intensify the mechanical stress in the bulk material [10]. For example, Li-ion battery cathodes consisting of nanosized doped $\mathrm{LiFePO}_{4}$ are documented to show a difference of approximately $7 \mathrm{mV}$ over a wide range of lithiation between the OCV measured after charge and discharge steps, respectively [15].

Beyond mechanical stress, thermodynamic effects are causing hysteresis in electrode potentials. The energetic favorable lithiation states are related to minima in the free energy of the individual particles of an electrode. The OCV hysteresis is attributed to the varying lithium insertion rates into the particles within a comprehensive electrode. The electrode potentials of a plurality of individual particles yield the resulting electrode potential being different from the progress of the potential of one single particle becoming lithiated. The potential blend of all the particles involved determines the potential of the entire electrode [16].

\section{Porous Electrode}

The electrodes of Li-ion batteries consist of metal foils (in the majority of cases, copper foil at the anode and aluminum

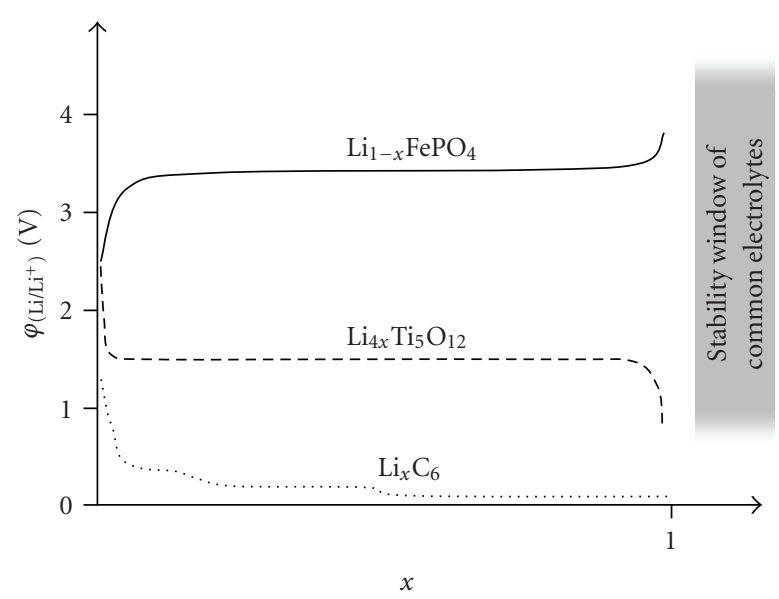

Figure 1: Potential against $\mathrm{Li} / \mathrm{Li}^{+}$of three different electrode materials, showing two-phase transition, plotted over the lithium content $x[12-14]$.

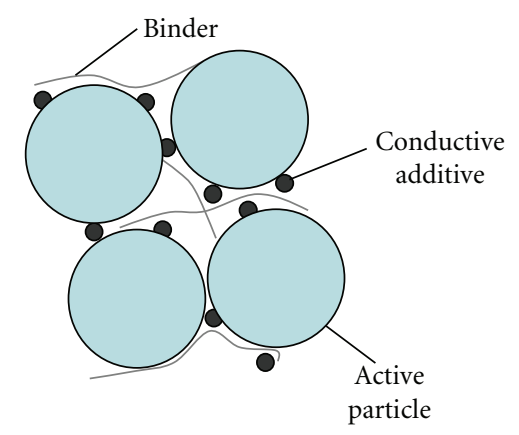

FIGURE 2: Illustration of a small part of an electrode, including active particles, binder, and conductive additives.

foil at the cathode side) coated with a composite including the actual active materials. Beside the active particles, the coating includes binder components (mostly polyvinylidene fluoride) and current conductive additives (e.g., carbon black, carbon nanotubes, etc.). The binder actually causes the mechanical stability of the coating. The conductive additives are mandatory to achieve a good electrical contact among the active particles and between the active material and the metal foils. In Figure 2, the 2D illustration of a small part of the composite electrode material is depicted schematically, where the binder material holds the active materials together, and conductive additives arrange the electric contact from particle to particle.

The electric conductivity of the conductive additives is orders of magnitudes higher than for the most cathodeactive materials; especially undoped $\mathrm{LiFePO}_{4}$ is almost an isolator [18]. Electron conduction within the active material is mainly associated to the lithium insertion/extraction process within the distinct particles. In order to achieve a low ohmic resistance for electron transportation, dopant agents (e.g., $\mathrm{W}, \mathrm{Mg}$, $\mathrm{Ti}$, and $\mathrm{Al}[18]$ ) are added to enhance the active materials' conductivity. Moreover, small-sized active (nano-) particles are incorporated to generate a high surface area and to realize short diffusion lengths inside 


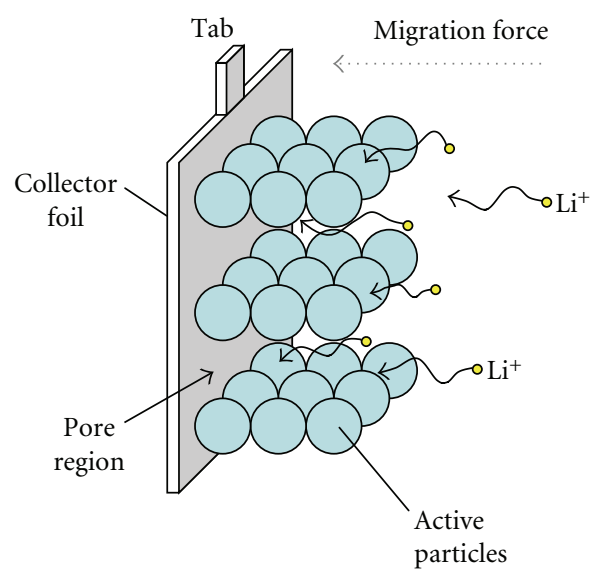

FIGURE 3: Illustration of a porous electrode geometry where lithium ions are inserted.

the active particles. Doping and the usage of small particles reduce electric losses within the active particles. Even the electric conductivity of the actual active material can be increased significantly, and the electron migration between the particles and the collector foils during load mainly occurs within the conductive additives. A high content of additives enables a good electron transport from the current collector towards the active particles and vice versa. However, the electric contact differs from particle to particles. Especially for nanoparticles contacting with additives or coating within conductive carbon layers it is a critical issue $[14,16]$. Hence, some particles have a better contact to the collector foil than other particles. Particles with a good electric contact are therefore favorable current paths during charge/discharging of the electrode, also yielding a nonuniform lithium insertion and extraction. Especially, during high current charging and discharging, the voltage drops along the various current paths have major impact on the $\mathrm{Li}$ insertion/extraction rate distribution among the active particle of an electrode.

Beside the electronic contact, the ionic contact at the interface between active material and the electrolyte is critical to achieve a good electrode performance. Therefore, smallsized active particles and a high porosity are preferable. High porosity yields a high amount of liquid electrolyte within the electrode and therefore generates a good ionic contact of the active particles. Furthermore, high porosity enables a fast ion exchange between the electrodes of a battery as more ions can migrate within in the wider pores of an electrode. But high porosity is counteracted by a lower energy density and an electronic contacting of the particles. So the optimum porosity, determined by calendering the coated foils, is a trade-off between the mentioned properties [19].

A simplified illustration of a porous electrode is depicted in Figure 3, where pore regions exist between the active particles (binder and conductive additives are not shown).

During Li insertion, the ions migrate from the counterelectrode towards the active particles where the insertion occurs. During $\mathrm{Li}$ insertion, the $\mathrm{Li}^{+}$ion concentration within the pores decreases as the ions are absorbed by the active material. To reach the inner particles, the ions propagate within the pores (migration and diffusion forced) from the electrode surface towards the inner regions. Due to Kohlrausch's law, the ion conductivity of the electrolyte faction within the pores decreases as the ion concentration decreases [20]. A lower ionic conductivity within the inner pore regions makes the $\mathrm{Li}$ insertion in the electrode's outermost particles more favorable, also yielding a nonuniform phase transformation among the particles. At high ion conduction rates, that is, high charge/discharge currents, the ion concentration variation along the pores depth increases, additionally forcing a more nonuniform Li insertion process among the particular particles.

As aforementioned, the hysteresis of two-phase transition materials (i.e., higher electrode potential against $\mathrm{Li} / \mathrm{Li}^{+}$after $\mathrm{Li}$ extraction than after $\mathrm{Li}$ insertion, at the same stoichiometric Li content) refers to microscopic effects within the particles and the distributed Li insertion/extraction among the particles. After low current rate SOC adjustment (e.g., to SOC $=50 \%$ ), the stoichiometric Li content of the particles can be assumed to be normally distributed, due to variations of particle sizes and variation of the electric/ionic contact of the individual particles, and therefore OCV hysteresis can be observed (e.g., in $\mathrm{LiFePO}_{4}$ electrodes $[15,16]$ ).

Through high-load application, the Li insertion/ extraction rates and therefore the Li contents within the particles cannot be assumed to be normally distributed, due to diffusion limitations within the porous electrode. The $\mathrm{Li}$ insertion in an electrode's outermost region proceeds significantly faster than the insertion into in the inner regions, as a consequence of ion depletion within the pores. The outermost particles become completely transformed into the lithiated state, and the inner particles remain almost delithiated. The strong $\mathrm{Li}$ content variance among the delithiated and lithiated particles yields an equalization ion exchange between the particles after a certain mid-SOC is adjusted. During this subsequent relaxation, the former almost delithiated inner particles become more lithiated, and the electrode's outer particles release Li. The process is equal to a case where almost one-half of the electrode is charged and the other part is discharged. Thus, after relaxation, the potential (against $\mathrm{Li} / \mathrm{Li}^{+}$) of the comprehensive electrode lies between the potential after Li insertion and the potential after Li extraction, respectively. Hence, the remaining hysteresis shrinks as the SOC is adjusted with higher currents, due to the stronger nonuniformity among the particles' Li contents. Measurements to validate the mentioned correlation on Li-ion cells including two-phase transition materials are documented in the following sections.

\section{Experimental}

Electrical tests on individual cells are done on a Digatron test bench $( \pm 6 \mathrm{~V})$. Tests are carried out on prototype Li-ion cells, including $\mathrm{LiFePO}_{4}$-based cathode and graphite-based anode. Both active materials are known to perform a twophase transition and comprise OCV hysteresis, as well.

To give insight to the OCV hysteresis characteristics of the encountered cells, full-cycle tests are carried out. The cells are fully charged through CCCV (constant current 
$0.5 \mathrm{C}$ (C-rate), constant voltage $3.6 \mathrm{~V}$ for 1 hour) charging regime $(\rightarrow \mathrm{SOC}=100 \%)$. After 30 minutes rest, the cells are gradually discharged in 5\% $\Delta$ SOC steps (according to the nominal capacity). Every discharge step a relaxation time of 3 hours (in open-circuit condition) is included. The gradual discharge is repeated until $100 \%$ of the nominal capacity is extracted. Then, the cells are gradually charged again (5\% $\triangle$ SOC steps, $0.5 \mathrm{C}$ current, 3 hours rest, included each step) until the cutoff voltage of $3.6 \mathrm{~V}$ is reached.

In order to investigate the impact of the load current rate on the occurrence and extent of the actual hysteresis, the cells' SOC $=50 \%$ is adjusted by charging of empty cells (SOC $=0 \%$, in relation to the cells' nominal capacities) and by discharging of completely charged cells (SOC $=100 \%$ ). The charge/discharge sequences are carried out with two different current rates. First, the target SOC $=50 \%$ is adjusted with $0.5 \mathrm{C}$. Thereafter, the cell individual open-circuit voltages are tracked for 8 hours. This test procedure is repeated with a current rate of $10 \mathrm{C}$ for adjusting an SOC $=50 \%$. Again, the OCV progresses are logged for 8 hours after the SOC adjustment is finished.

During testing, the cells under investigation are placed in a climate chamber from CTS, and the cells' ambient temperatures are constantly held at $25^{\circ} \mathrm{C}$.

\section{Results and Discussion}

Through gradual discharging and subsequent charging of single cells, the relation between SOC and OCV is obtained. A typical progress of the OCV, measured after 3-hour relaxation time, plotted over the distinct SOC values, is illustrated in Figure 4.

Two facts are obvious from the shown results. First, the OCV after the certain discharge steps (marked with $\left.\mathrm{OCV}_{\text {discharge }}\right)$ is significantly lower than the OCV after charging $\left(\mathrm{OCV}_{\text {charge }}\right)$. Hence, two curves exist, enclosing a hysteresis. The voltage gap between both curves depends on the SOC, where a maximum gap of approximately $40 \mathrm{mV}$ can be found at SOC $=25 \%$. Second, the OCV curves comprise significant plateaus and edges referring to the edges and plateaus of the graphite anode (see Figure 1). In the illustrated curves, the potential edge related to the stoichiometric state $\mathrm{Li}_{0.5} \mathrm{C}_{6}$ lies in the range $\mathrm{SOC}=65-75 \%$ of the investigated prototype cells. Hence, the increasing gradient towards the fully charged state, is attributed to the potential of the $\mathrm{LiFePO}_{4}$-based cathode. Near to the fully charged state the hysteresis voltage gap decreases, but it is still apparent in the tested case, after 3 hours of rest. Due to the long rest periods in between each charge/discharge step and from this first test, the OCV hysteresis can be assumed to be stable, at least from the practical point of view.

To give further insight on how the current rate influences the extent of the OCV hysteresis, the second OCV tests is carried out. The progress of the measured cell voltages after the SOC $=50 \%$ is adjusted through charging and discharging (with $0.5 \mathrm{C}$ and $10 \mathrm{C}$, resp.) is depicted in Figure 5.

Obviously, the OCV values after 8-hour relaxation time differ significantly, even if the SOC is exactly the same in all the four given cases. The OCV difference after low-current

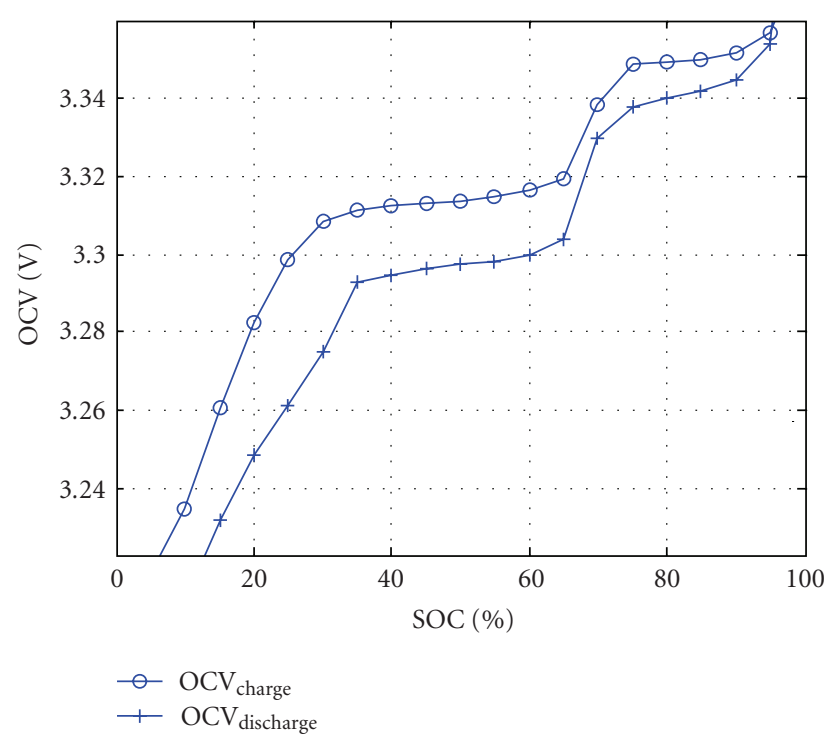

FIGURE 4: OCV after 3 hours rest plotted over the SOC, after stepwise discharging and charging with $0.5 \mathrm{C}$.

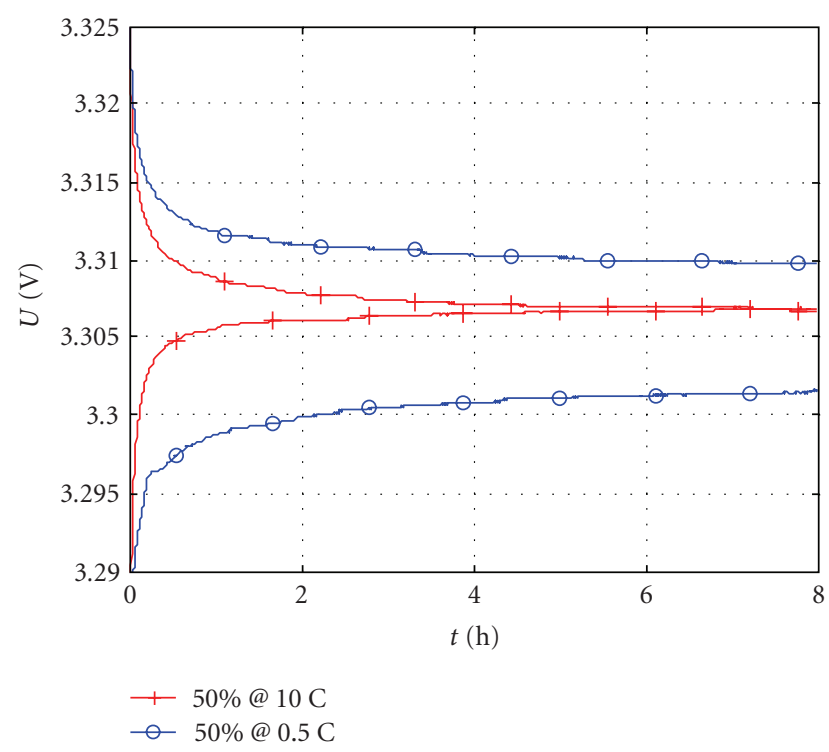

FIGURE 5: Progress of the cell voltage during rest over 8 hour, where the SOC $=50 \%$ is adjusted through charging and discharging with various current rates.

load is approximately $8 \mathrm{mV}$, whereas no difference remains as $\mathrm{SOC}=50 \%$ is adjusted with $10 \mathrm{C}$, being independent from the current direction. Noticeably, the OCV hysteresis has completely vanished after high-load application. Herein, the relaxation takes approximately 5 hours until the voltages have completely converged. After shorter rest durations, a slight OCV hysteresis would be observed.

In comparison to the voltage gap between charge and discharge OCV curve at SOC $=50 \%$ of approximately $18 \mathrm{mV}$ in Figure 4 (gradual cycle test), the gap after $0.5 \mathrm{C}$ load (one charge/discharge step to $\mathrm{SOC}=50 \%$ ) is only $11 \mathrm{mV}$. Hence, the higher mean current during SOC adjustment in one 
straight step $(0.5 \mathrm{C})$ in comparison to the OCV test with gradual steps $(0.5 \mathrm{C}$ with 3 hours of rest every $5 \% \Delta \mathrm{SOC}$ step) already yields a reduced voltage hysteresis.

On one hand, the test results confirm the hypothesis outlined in Section 3. Interparticle charge transfer, resulting from strong inhomogeneities among the particles' lithium contents, is a reasonable explanation for a shrinking OCV hysteresis with increasing current application.

On the other hand, the results show that the relaxation takes several hours (at room temperature) and therefore proceeds very slowly, giving a hint that the occurring processes are attributed to very slow solid-state diffusion processes from particle to particle. Even the voltages after low-current SOC adjustment are drifting for approximately 5 hours until a steady state is reached. Hence, at temperatures below zero degrees, the relaxation is supposed to take even more time than in the presented cases, due to the decreasing diffusion constants at low temperatures. Thus, stepwise OCV measurements at low temperature on Li-ion cell and even half cells should include rest periods of several hours to achieve reliable results. But this aspect has not been completely enlightened yet.

The impact of the current rate on the occurrence of OCV hysteresis and the very slow OCV recovery are critical for commonly used model-based battery state estimation methods. Evaluating battery cells' OCV values is in many cases a reliable way to estimate the SOC-in the shown cases it would not. The presented results point out that for Liion cells including two-phase transition active materials, long rest periods are to be considered to evaluate the OCV in order to achieve the instantaneous SOC. Moreover, methods are mandatory to reconstruct the impact of the current rate on the occurrence and extent of the actual hysteresis voltage. For implementation in battery management systems, physical models may be too complex, being not suitable for a fast realtime calculation. Exert systems and artificial neural network (ANN) will be more promising. However, the development of one of the latter-mentioned systems, operating reliably under every operation condition, lies beyond the scope of this work and is therefore left out here.

The results given in this section are based on measurements on a certain type of Li-ion cells including $\mathrm{LiFePO}_{4}$ based cathode and graphite anode. For lithium titanate $\left(\mathrm{Li}_{4} \mathrm{Ti}_{5} \mathrm{O}_{12}\right)$, also performing a two-phase transition over a wide SOC range, hysteresis effects are not documented yet. However, $\mathrm{Li}$-ion cells including $\mathrm{LiFePO}_{4}$ cathodes and $\mathrm{Li}_{4} \mathrm{Ti}_{5} \mathrm{O}_{12}$ anodes can even though show pronounced OCV hysteresis, due to the cathode characteristics. Hence, investigations on the OCV hysteresis of the latter-stated cell technology are a topic of our future research.

\section{Conclusion}

The current intensity during load of Li-ion battery cells, including two-phase transition active materials, influences the $\mathrm{Li}$ insertion/extraction rate among the particles of the electrodes. High current rates are assumed to yield strong inhomogeneities in the particles' lithium contents and therefore induce an ion transfer from lithiated particle towards delithiated particles. The test results show that high current load affects the macroscopic processes in a way that OCV hysteresis vanishes for Li-ion cells, which are regularly showing OCV hysteresis after low current application.

To quantify and reconstruct the illustrated effects under various conditions new methods are to be encountered, considering the load current intensity besides the SOC, the temperature, and the load current direction as factors influencing the OCV hysteresis.

\section{References}

[1] M. Thele, O. Bohlen, D. U. Sauer, and E. Karden, "Development of a voltage-behavior model for NiMH batteries using an impedance-based modeling concept," Journal of Power Sources, vol. 175, no. 1, pp. 635-643, 2008.

[2] K. P. Ta and J. Newman, "Proton intercalation hysteresis in charging and discharging nickel hydroxide electrodes," Journal of the Electrochemical Society, vol. 146, no. 8, pp. 2769-2779, 1999.

[3] J. Barker, R. Pynenburg, R. Koksbang, and M. Y. Saidi, "An electrochemical investigation into the lithium insertion properties of $\mathrm{Li}_{x} \mathrm{CoO}_{2}$," Electrochimica Acta, vol. 41, no. 15, pp. 2481-2488, 1996.

[4] A. K. Sleigh, J. J. Murray, and W. R. McKinnon, "Memory effects due to phase conversion and hysteresis in $\mathrm{Li} / \mathrm{Li}_{x} \mathrm{MnO}_{2}$ cells," Electrochimica Acta, vol. 36, no. 9, pp. 1469-1474, 1991.

[5] D. Carlier, I. Saadoune, M. Ménétrier, and C. Delmas, "Lithium electrochemical deintercalation from $\mathrm{O} 2-\mathrm{LiCoO}_{2}$ : structure and physical properties," Journal of the Electrochemical Society, vol. 149, no. 10, pp. A1310-A1320, 2002.

[6] M. D. Levi and D. Aurbach, "Frumkin intercalation isotherma tool for the description of lithium insertion into host materials: a review," Electrochim Acta, vol. 45, pp. 167-185, 1999.

[7] C. V. Ramana, A. Mauger, F. Gendron, C. M. Julien, and K. Zaghib, "Study of the Li-insertion/extraction process in $\mathrm{LiFePO}_{4} / \mathrm{FePO}_{4}$," Journal of Power Sources, vol. 187, no. 2, pp. 555-564, 2009.

[8] L. Laffont, C. Delacourt, P. Gibot et al., "Study of the $\mathrm{LiFePO}_{4}$ / $\mathrm{FePO}_{4}$ two-phase system by high-resolution electron energy loss spectroscopy," Chemistry of Materials, vol. 18, no. 23, pp. 5520-5529, 2006.

[9] J. Goodenough, A. Padhi, K. S. Nanjundaswamy, and C. Masquelier, "Cathode materials for secondary (rechargeable) lithium batteries," US patent 5910382, 1999.

[10] N. Meethong, H. Y. S. Huang, S. A. Speakman, W. C. Carter, and Y. M. Chiang, "Strain accommodation during phase transformations in olivine-based cathodes as a materials selection criterion for high-power rechargeable batteries," Advanced Functional Materials, vol. 17, no. 7, pp. 1115-1123, 2007.

[11] M. A. Roscher, J. Vetter, and D. U. Sauer, "Characterisation of charge and discharge behaviour of lithium ion batteries with olivine based cathode active material," Journal of Power Sources, vol. 191, no. 2, pp. 582-590, 2009.

[12] M. Winter, J. O. Besenhard, M. E. Spahr, and P. Novák, "Insertion electrode materials for rechargeable lithium batteries," Advanced Materials, vol. 10, no. 10, pp. 725-763, 1998.

[13] N. Meethong, H.-Y. Shadow Huang, C. Crater, and Y.-M. Chiang, "Size-dependent lithium miscibility gap in nanoscale $\mathrm{Li}_{1-x} \mathrm{FePO}_{4}$, " Electrochemical and Solid-State Letters, vol. 10, pp. A134-A138, 2007. 
[14] G. J. Wang, J. Gao, L. J. Fu, N. H. Zhao, Y. P. Wu, and T. Takamura, "Preparation and characteristic of carbon-coated $\mathrm{Li}_{4} \mathrm{Ti}_{5} \mathrm{O}_{12}$ anode material," Journal of Power Sources, vol. 174, no. 2, pp. 1109-1112, 2007.

[15] N. Meethong, Y.-H. Kao, S. Speakman, and Y.-M. Chiang, "Structural and electrochemical studies of aliovalent cation doped olivines," in Proceedings of the Pacific Rim Meeting on Electrochemical and Solid-State Science, Honolulu, Hawaii, USA, 2008.

[16] W. Dreyer, J. Jamnik, C. Guhlke, R. Huth, J. Moškon, and M. Gaberšček, "The thermodynamic origin of hysteresis in insertion batteries," Nature Materials, vol. 9, no. 5, pp. 448-453, 2010.

[17] U. S. Kasavajjula, C. Wang, and P. E. Arce, "Discharge model for $\mathrm{LiFePO}_{4}$ accounting for the solid solution range," Journal of the Electrochemical Society, vol. 155, no. 11, pp. A866-A874, 2008.

[18] M. Thackeray, "Lithium-ion battries: an unexpected conductor," Nature Materials, vol. 1, no. 2, pp. 81-82, 2002.

[19] C. Fongy, A. C. Gaillot, S. Jouanneau, D. Guyomard, and B. Lestriez, "Ionic vs electronic power limitations and analysis of the fraction of wired grains in $\mathrm{LiFePO}_{4}$ composite electrodes," Journal of the Electrochemical Society, vol. 157, no. 7, pp. A885-A891, 2010.

[20] F. W. G. Kohlrausch, Das Leitvermögen der Elektrolyte, Insbesondere der Lösungen, Taschenbuch, BiblioBazaar, Charleston, SC, USA, 2009. 


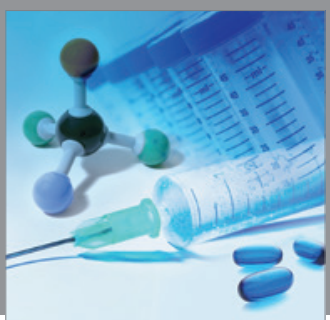

International Journal of

Medicinal Chemistry

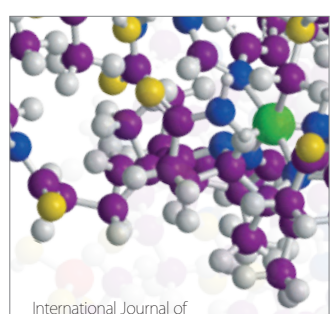

Carbohydrate Chemistry

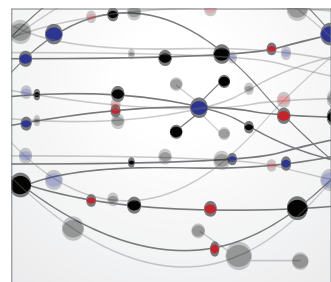

The Scientific World Journal
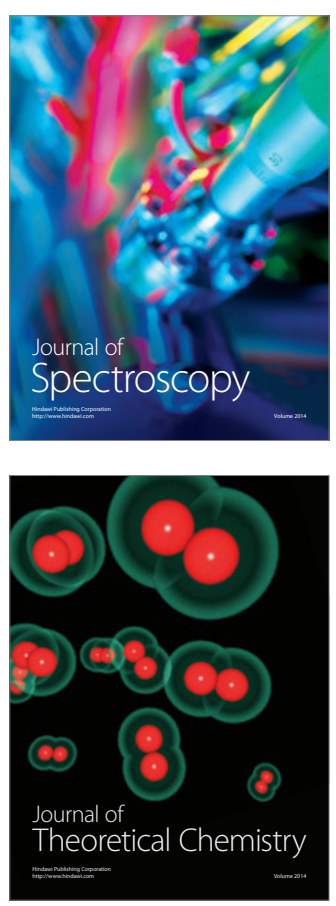
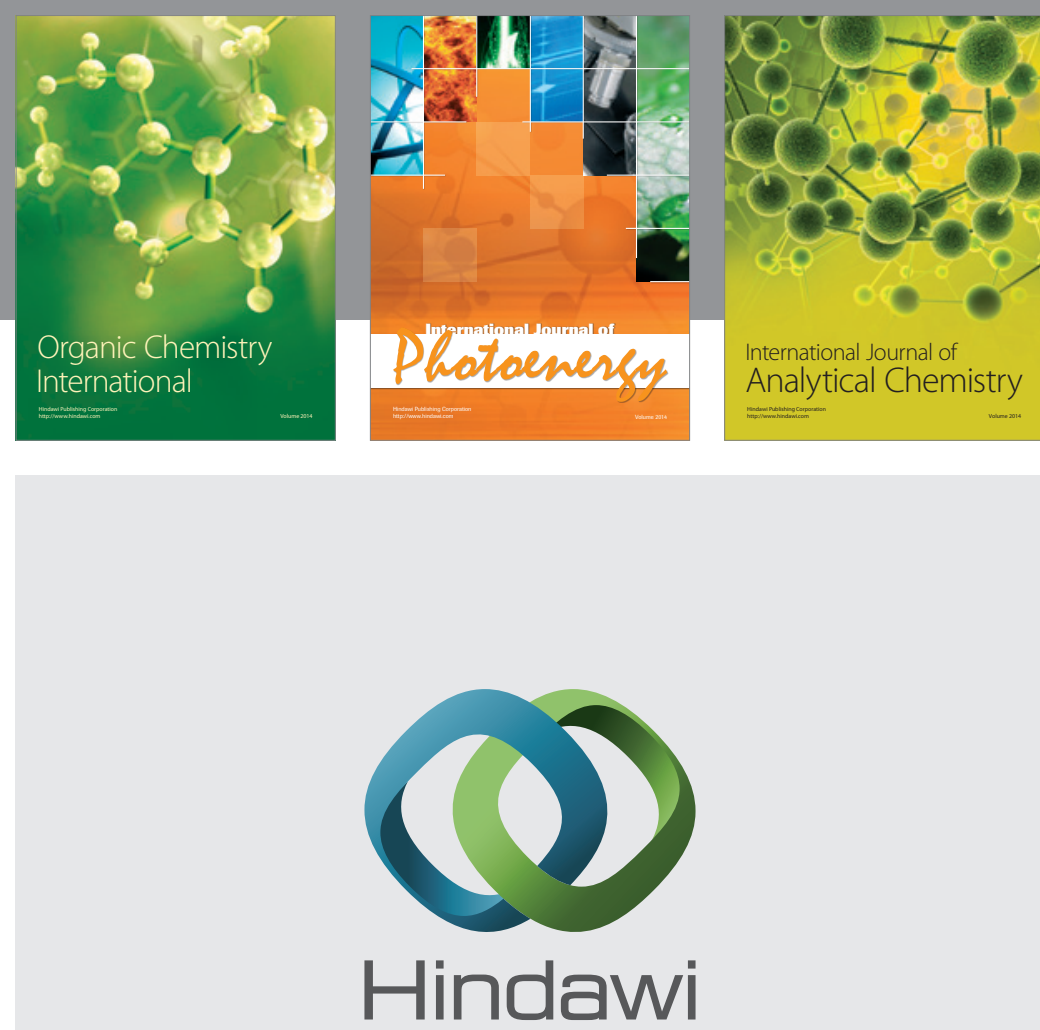

Submit your manuscripts at

http://www.hindawi.com
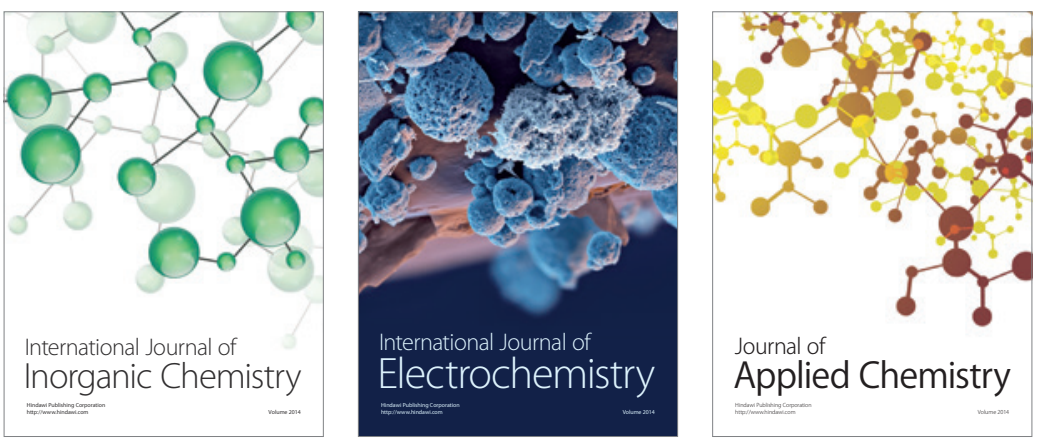

Journal of

Applied Chemistry
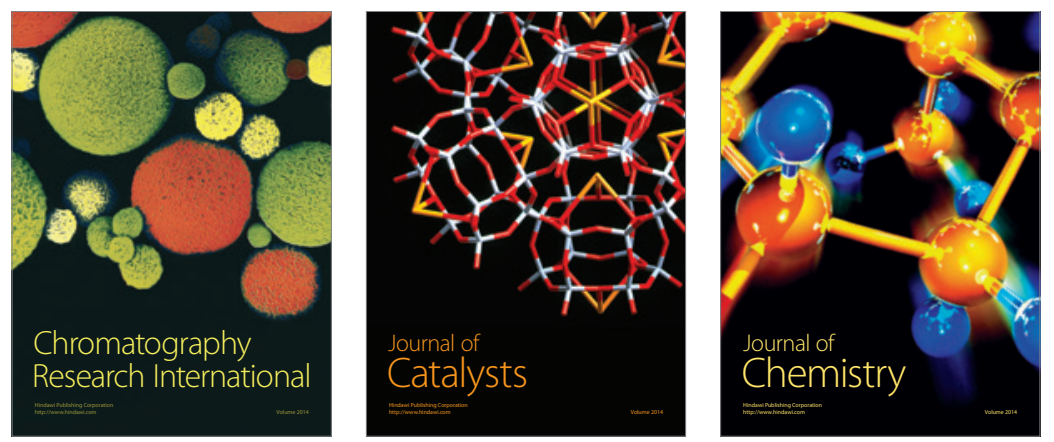
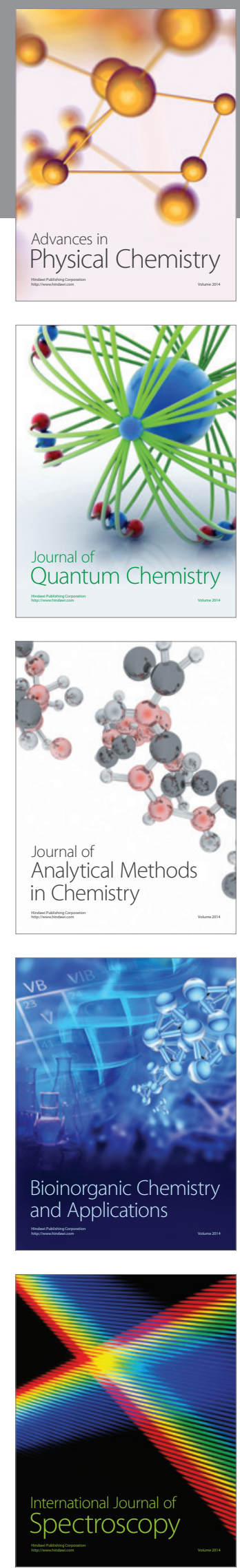